\title{
On Wave Splitting, Source Separation and Echo Removal with Absorbing Boundary Conditions
}

D. Baffet and M. J. Grote 


\title{
On Wave Splitting, Source Separation and Echo Removal with Absorbing Boundary Conditions
}

\author{
Daniel Baffet, Marcus J. Grote \\ Department of Mathematics and Computer Science, \\ University of Basel, \\ Spiegelgasse 1, \\ 4051 Basel, Switzerland
}

September 27, 2018

\begin{abstract}
Starting from classical absorbing boundary conditions (ABC), we propose a method for the separation of time-dependent wave fields given measurements of the total wave field. The method is local in space and time, deterministic, and makes no prior assumptions on the frequency spectrum and the location of sources or physical boundaries. By using increasingly higher order ABC, the method can be made arbitrarily accurate and is, in that sense, exact. Numerical examples illustrate the usefulness for source separation and echo removal.
\end{abstract}

\section{Introduction}

For decades absorbing boundary conditions ${ }^{1}$ (ABC) have been used to truncate computational domains $[2,5,11,10,6,13]$ for the simulation of time-dependent wave phenomena in

\footnotetext{
${ }^{1}$ a.k.a. nonreflecting, transparent, outgoing, one-way, etc. boundary conditions
}

unbounded regions. Typically, an ABC consists of a linear partial differential "one-way" operator, $B$, which eliminates outgoing waves. By imposing the boundary condition

$$
B[u]=0
$$

at the outer artificial boundary, (unphysical) incoming waves are set to zero while outgoing waves remain unaffected. The result is a boundary condition that completes the statement of a well posed initial boundary value problem (IBVP) and allows outgoing waves to leave the computational domain without spurious reflections.

Here we show that one-way operators also permit to split and recover individual wave fields given observations from a time-dependent total wave field, a problem that commonly arises in a variety applications under different disguise. During the collection of marine hydrophone seismic data, for instance, sound wave reflected from the sea floor are recorded by hydrophone streamers towed at a finite depth. After reflection from the (moving) ocean surface, however, 
those sound waves travel back generating an (unwanted) echo; the process of removing that ghost signal from the data is also known as "deghosting" [16]. In signal processing, blind source separation uses ray-based statistical tools to detect individual sources [3] or remove noise [1] from their recorded mixture.

When an unknown medium or obstacle is illuminated by an incident probing wave, the scattered data recorded at remote sensors can be used to recover the nature, location or shape of the buried object. While many methods exist to solve such inverse scattering problems, they always assume that the scattered field is readily available by subtraction of the incident wave from total field measurements. Although essential for any subsequent inversion, the extraction of the scattered field certainly becomes nontrivial if the location, spatial distribution or time dependence of the original source are not precisely known or other undesired sources or physical boundaries interfere with the signal. In transcranial ultrasonic imaging [14], for instance, the time signature of the small shock wave induced by cavitation bubbles is never precisely known. Similarly, in photoacoustic imaging [17], the time signature of the laser induced ultrasonic pressure wave generated by the transient thermoelastic expansion of biological tissues is hardly available.

In the presence of two or more obstacles, the inversion from total field measurements is greatly simplified if the multiple scattered fields can be split into individual outgoing components; then, each isolated scattering problem will be smaller in size and less ill-conditioned than their total sum. There is a long history of wave splitting techniques for multiple scattering problems, but mainly in the frequency domain. By combining the inverse Radon approximation with a Galerkin ansatz, Griesmaier,
Hanke and Sylvester determine the convex scattering support of individual far-field components separately [7]. For time-dependent source separation, Potthast, Fazi and Nelson recently devised a filter using the point source method via Fourier transform in the frequency domain [15]. Recently, Grote, Kray, Nataf and Assous devised a method to split a time-dependent, scattered, total wave field into its distinct outgoing components induced by separate sources or obstacles [9]. While their approach is purely local in space and time, it still requires deriving a first-order hyperbolic PDE satisfied by each scattered field component on the observational boundary.

Here we propose a different approach for the separation of time-dependent wave fields, which is still local in space and time but does not require deriving any new PDE. In Section 2, we consider a simple generic set-up to illustrate the basic underlying idea. Next, we recall in Section 3 a particular family of one-way operators based on the high-order ABC by Collino [4]. Finally, in Section 4, we illustrate through numerical examples how to apply the our one-way wave splitting approach to source separation and echo removal.

\section{Wave splitting}

We consider the simple but generic set-up of source separation in free space to present the main idea underlying our approach for wave splitting. Hence, let the total wave field $u$ satisfy the wave equation

$$
\frac{\partial^{2} u}{\partial t^{2}}-\Delta u=F(x, y, t)
$$

in $\mathbb{R}^{2} \times(0, T)$ with homogeneous initial conditions at time $t=0$. Now, let $F$ be given by

$$
F=F_{1}+F_{2}
$$




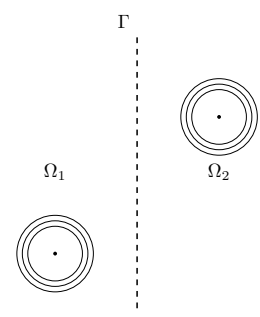

Figure 1: Source separation, generic set-up. Left: unsplit problem posed in $\mathbb{R}^{2}$; right: setup in $D_{\infty}$ for the approximation $v_{1}$ of $u_{1}$

where each $F_{1}$ and $F_{2}$ is compactly supported in $\Omega_{1}=\{x<0\}$, and $\Omega_{2}=\{x>0\}$, respectively. Then, the wave field $u$ admits the unique (Kirchhoff) decomposition [8]

$$
u=u_{1}+u_{2},
$$

where $u_{1}$ and $u_{2}$ each solve $(2)$ in $\mathbb{R}^{2} \times(0, T)$ with $F=F_{1}$ and $F=F_{2}$, respectively. From $\Omega_{1}$, the wave field $u_{1}$ thus appears purely outgoing as it crosses the separating artificial boundary $\Gamma(y$ axis) at $x=0$, and vice-versa for $u_{2}$.

More generally, when $\Omega$ includes nonzero initial conditions, obstacles or inhomogeneities, all compactly supported outside some neighborhood of $\Gamma$, the same decomposition (4) still holds in that neighborhood. Each wave field $u_{i}$ then solves the homogeneous wave equation with zero initial conditions, no obstacles and constant wave speed outside $\Omega_{i}, i=1,2$; therefore, $u_{i}$ is entirely determined inside $\Omega_{j}, j \neq i$, by the boundary condition imposed on $\Gamma$.

Given the time-dependent total wave field $u$ and its normal derivative $\partial u / \partial n$ on $\Gamma$, we wish to recover $u_{1}$ and $u_{2}$ on $\Gamma$ for all $t$. As seen from $\Gamma, u_{1}$ is purely rightward moving whereas $u_{2}$ is purely leftward moving; therefore, we may use one-way operators to distinguish between them.
Let $B$ be a one-way operator such that $B\left[u_{2}\right]=0$ on $\Gamma$ and the corresponding IBVP in $\Omega_{2}$ is well-posed. Applying $B$ to $u$ on $\Gamma$ then yields

$$
B\left[u_{1}\right]=B\left[u_{1}\right]+B\left[u_{2}\right]=B[u] .
$$

Assuming that $B[u]$ can be computed from the measured total field $u$, (5) clearly yields an inhomogeneous boundary condition for $u_{1}$. Still, it is by no means obvious how to reconstruct $u_{1}$ itself from (5), as it involves not only time and tangential but also normal derivatives of $u_{1}$ at $\Gamma$. For $\Gamma$ a circle, (5) can be used to derive a hyperbolic partial differential equation for $u_{1}$, which involves only time and tangential derivatives and thus can be solved on $\Gamma[9]$.

Instead, we note that $u_{1}$ is the unique solution of the IBVP in $D_{\infty}=\{x>0\}$ :

$$
\begin{aligned}
\frac{\partial^{2} v}{\partial t^{2}}-\Delta v & =0 & & \text { in } D_{\infty} \times(0, T) \\
B[v] & =g(y, t) & & \text { on } \Gamma \times(0, T) \\
v(0) & =0 & & \text { in } D_{\infty},
\end{aligned}
$$

with $g=B[u]$, which is well-posed by assumption. Although here $D_{\infty}$ coincides with $\Omega_{2}$, this is not true in general - see Section 4.2, for instance. To recover $u_{1}$, we simply solve numerically the IBVP (6) in $D_{\infty}$. In fact, when $u_{1}$ is only required on $\Gamma$, we may simply restrict the computation to the vicinity of $\Gamma$. Clearly, once $u_{1}$ is known we immediately recover $u_{2}=u-u_{1}$.

In practice, the $\mathrm{ABC}(1)$ does not eliminate all rightward moving waves. Then $B\left[u_{2}\right]$, albeit small, is not identically zero and the first equality in (5) holds only approximately. Nevertheless, we may still solve (6) with $g=B[u]$, thereby introducing a perturbation, $B\left[u_{2}\right]$, into $g$. Since (6) is well-posed, that perturbation will only result in a small error in the reconstruction 
of $u_{1}$. Depending on the application considered, that error may or may not be significant. By choosing a sufficiently accurate one-way operator $B$, however, that error can always be made arbitrarily small.

\section{High-order ABC}

To recover $u_{1}$ from $u$ on $\Gamma$, we require a one-way operator $B$ that discriminates between incoming and outgoing waves and also yields a well-posed IBVP (6) in $D_{\infty}$. Here, we consider the highorder $\mathrm{ABC}$ by Collino $[4,6]$ and let $B$ equal the corresponding $P$-th order one-way operator:

$$
B[w]=\left.\left(\frac{\partial w}{\partial t}-\frac{\partial w}{\partial x}\right)\right|_{\Gamma}-\sum_{p=1}^{P} b_{p} \frac{\partial \psi_{p}}{\partial t}
$$

where $\left.v\right|_{\Gamma}$ denotes the restriction of $v$ to $\Gamma$ and each auxiliary function $\psi_{p}=\psi_{p}(y, t), p=$ $1, \ldots, P$, solves the following initial value problem:

$$
\begin{aligned}
& \frac{\partial^{2} \psi_{p}}{\partial t^{2}}-a_{p} \frac{\partial^{2} \psi_{p}}{\partial y^{2}}=\frac{\partial^{2} w}{\partial y^{2}}(x, y, t), \quad(x, y) \in \Gamma, \\
& \psi_{p}(y, 0)=\frac{\partial \psi_{p}}{\partial t}(y, 0)=0
\end{aligned}
$$

Note that (7b) only involves tangential and time derivatives of $\psi_{p}$, which are only defined and computed on $\Gamma$.

For well-posedness [18], the parameters $a_{1}, \ldots, a_{P}$ and $b_{1}, \ldots, b_{P}$ must satisfy $0<a_{p}<1$ and $b_{p}>0$ for $p=1, \ldots, P, a_{p} \neq a_{k}$ for $p \neq k$, and

$$
\sum_{p=1}^{P} \frac{b_{p}}{1-a_{p}}<1 .
$$

Following [6], we choose

$$
\begin{aligned}
& a_{p}=\cos ^{2}\left(\frac{\pi p}{2 P+1}\right) \\
& b_{p}=\frac{2}{2 P+1} \sin ^{2}\left(\frac{\pi p}{2 P+1}\right) .
\end{aligned}
$$

Note that parameter values may be adapted to any particular problem. The ABC becomes increasingly accurate with increasing $P$; hence, it is exact in the sense that we can always choose $P$ sufficiently large to reduce the error in the boundary condition below a prescribed error tolerance (without moving $\Gamma$ any farther).

For computations, we truncate $D_{\infty}$ by a vertical perfectly matched layer (PML) in the $x$ direction [12]. In principle, the truncated domain (excluding the PML) can be arbitrarily narrow and even consist only of a fixed number of mesh points in width; thus, its width may even shrink with decreasing mesh size $h$.

\section{Numerical examples}

Here we consider two distinct applications of wave splitting: source separation and echo removal. All computations are performed on an equidistant Cartesian mesh with standard second-order finite differences in space and time. We let $T$ denote the final time, $h>0$ the mesh size and $\kappa>0$ the time step. The relative global error in the reconstruction $u_{1}^{h}$ of $u_{1}$ at $\Gamma$ is measured as

$$
E=\frac{\left\|u_{1}^{h}-u_{1}\right\|_{\Gamma, T}}{\left\|u_{1}\right\|_{\Gamma, T}},
$$

where

$$
\|w\|_{\Gamma, T}=\max _{n, j}|w|_{\Gamma}\left(y_{j}, t_{n}\right) \mid .
$$

for any grid function $w$ evaluated at time $t_{n}=$ $n \kappa, n \geq 0$. 


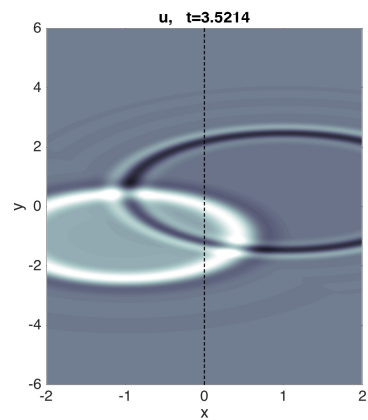

(a)

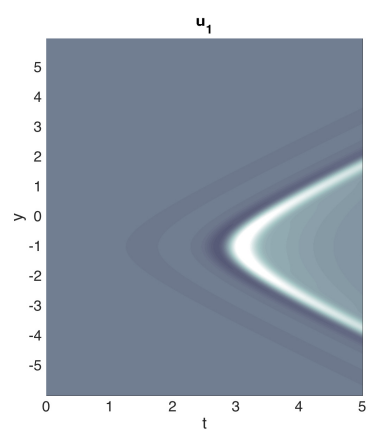

(c)

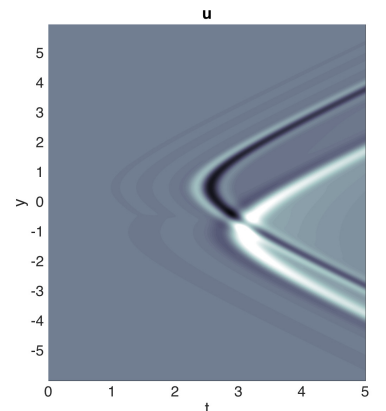

(b)

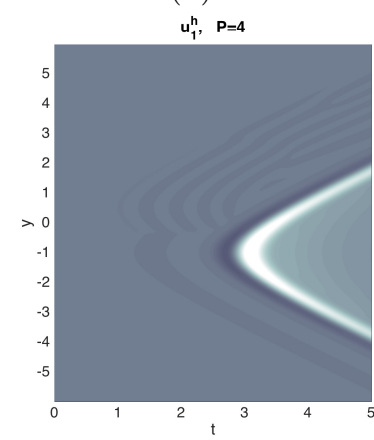

(d)
Figure 2: Source separation. (a) Snapshot of $u$ at $t \approx 3.5$; the measurement surface $\Gamma$ is marked by a dashed line, (b) the space-time data $u$ on $\Gamma$, (c) the reference solution $u_{1}$ (d) the split data $u_{1}^{h}$ obtained with $P=4$

\subsection{Source separation}

First, we consider the generic set-up from Section 2 and let the total wave field $u$ and its normal derivative $\partial u / \partial x$, both recorded at $\Gamma$, consist of two outward propagating circular waves $u=u_{1}+u_{2}$ in free space, each $u_{i}$ originating from its respective half-space $\Omega_{i}$ on either side of the $y$-axis. Following the approach described in Section 2, we shall now reconstruct $u_{1}$, given the time history of the total wave field $u$, and its normal derivative $\partial u / \partial x$ on $\Gamma$. To do so, we solve the IBVP (6) with $g=B[u]$ computed at $\Gamma$. The infinite domain $D_{\infty}$ is truncated at $x=5 h$, that is at a distance of five mesh points from $\Gamma$, beyond which a PML is added to absorb outward going waves. We set $h=10^{-2}$ and $\kappa>0$ according to the CFL stability condition of the leap-frog method.

Figure 2a shows a snapshot of the total field $u$ at $t \approx 3.5$ whereas Figure $2 \mathrm{~b}$ displays the space-time data on $\Gamma$ used for the reconstruction. In particular, we observe the emergence of two space-time cones as the leftward and rightward moving wave fronts cross $\Gamma$. The numerical solution, $u_{1}^{h}$, of the IBVP (6) is shown in Figure $2 \mathrm{~d}$ and compares remarkably well with the reference solution, $u_{1}$, shown in Figure $2 \mathrm{c}$. In fact, $u_{1}$ and $u_{1}^{h}$ essentially coincide, as shown in the left frame of Figure 3 where we compare their time evolution at a fixed location $(x, y)=(0,0)$. In the right frame of Figure 3, we observe that the relative space-time global error $E$, given by (10), decays exponentially with $P$ until it saturates at the level of the discretization error.

We recall that our wave-splitting approach makes no use of the locations or space-time dependence of the sources. In fact, it is independent of the sources generating the waves and would remain identical in the presence of obstacles or inhomogeneities.

\subsection{Echo removal}

Next, we show how to remove an (undesired) echo due to a physical boundary (wall, ocean surface, etc.) from the total wave field observed again on the $y$-axis $\Gamma$ at $x=0$. The incident wave field $u_{1}$ originates from the left half-space $\Omega_{1}=\{x<0\}$. After crossing the observational boundary $\Gamma$, the incident signal $u_{1}$ enters the 

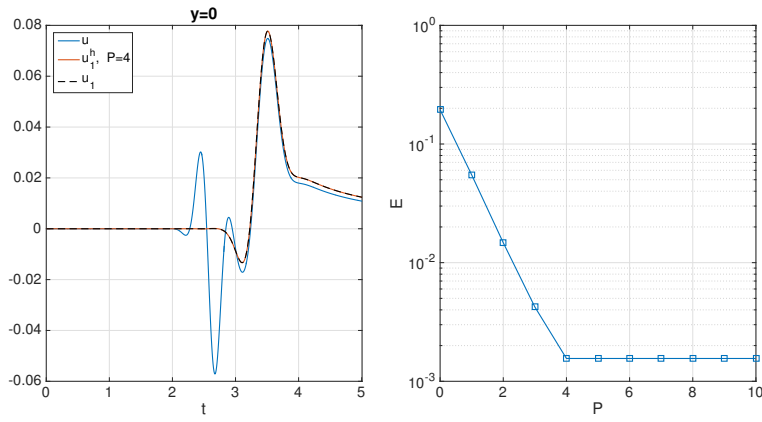

Figure 3: Source separation. Left: the numerical solution $u$ driven by the two sources, the reference solution $u_{1}$ and the reconstructed signal $u_{1}^{h}$ at $(x, y)=(0,0)$; right: the relative global error $E$ as a function of $P$.

subdomain $\Omega_{2}=\{0<x<b\}$ until it impinges upon the physical boundary $\partial \Omega=\Gamma_{\mathrm{BC}}$, located at $x=b$, where it is reflected; the resulting signal $u_{2}$ then propagates back and causes an (undesired) echo in the observations of the total field $u$ at $\Gamma$.

Hence, the total wave field $u$ satisfies (2) in $\Omega=(-\infty, b) \times \mathbb{R}$, with $b>0$ and homogeneous initial conditions at $t=0$. The source $F$ consists of two point sources located inside $\Omega_{1}$ and we impose a homogeneous Dirichlet boundary condition $u=0$ at the physical boundary $\partial \Omega=\Gamma_{\mathrm{BC}}$ located at $x=b$, for simplicity - see Figure 4 .

Given measurements of the total field at $\Gamma=$ $\{x=0\}$, we wish to recover the signal $u_{1}$ on $\Gamma$ from the total field $u$ and thus effectively remove the echo $u_{2}$ generated by the physical boundary at $x=b$. To do so, we let $D_{\infty}=\{x>0\}$ and consider (6), where $B$ is a Collino operator (7) of order $P$. Now, we again solve the IBVP (6) for $u_{2}$ numerically using the same parameters (mesh size, time-step, etc.) as in Section 2.1.

Figure 5a shows a snapshot of the total field

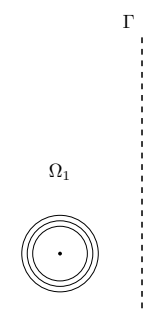

$$
\begin{array}{lll} 
& \multicolumn{1}{c}{\Gamma_{\mathrm{BC}}} \\
& & \\
& & \\
& & \\
& & \\
& & \\
& &
\end{array}
$$

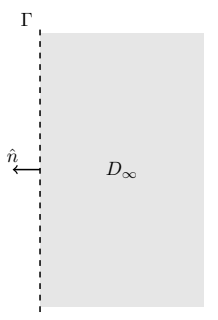

Figure 4: Echo removal, generic setup. Left: unsplit problem posed in $\mathbb{R}^{2}$; right: setup in $D_{\infty}$ for the approximation $v_{1}$ of $u_{1}$

$u$ at $t \approx 3.5$ whereas Figure $5 \mathrm{~b}$ displays the space-time data on $\Gamma$ used for the reconstruction. In Fig. 5b, in particular, we see the two space-time cones which correspond to the two point sources located at $(x, y)=(-0.5,0)$, and $(x, y)=(-0.7,0.5)$, respectively. Upon comparison of the total field $u$ in Figure $5 \mathrm{~b}$ with the reference solution $u_{1}$ in Figure $5 \mathrm{c}$, we observe how the reflection from the physical right wall propagates back and crosses the incoming signal while causing destructive interference behind the first arrival.

The numerical solution, $u_{1}^{h}$ of the IBVP (6) is shown in Figure 5d and compares remarkably well with the reference solution $u_{1}$. Again, $u_{1}$ and $u_{1}^{h}$ essentially coincide, as shown in the left frame of Figure 6 where we compare their time evolution at a fixed location $(x, y)=(0,0)$. In the right frame of Figure 6, we observe that the relative space-time global error $E$, given by (10), decays exponentially with $P$ until it saturates at the level of the discretization error.

Again, we emphasize that our wave-splitting approach does not rely on any information from the sources and applies regardless of the type of boundary condition imposed at the (possibly moving) physical boundary $\partial \Omega$. It would also 


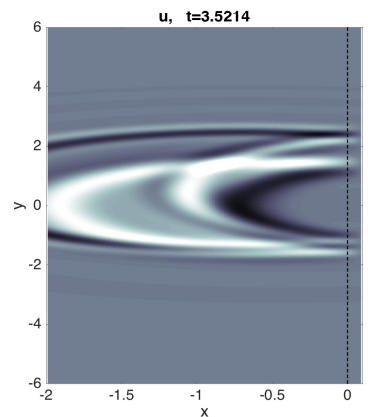

(a)

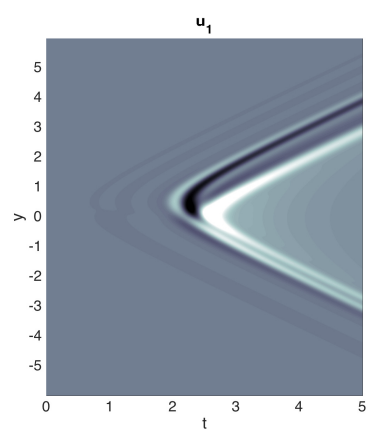

(c)

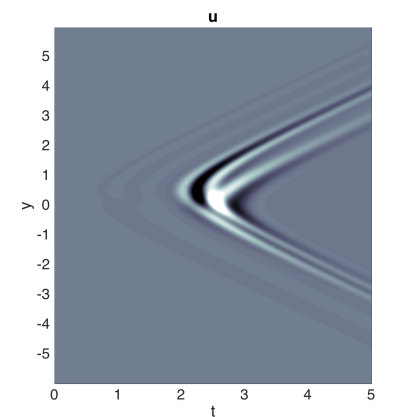

(b)

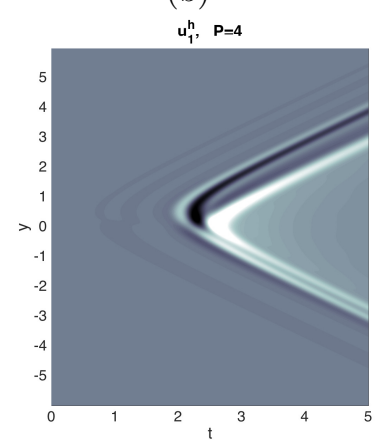

(d)

Figure 5: Echo removal. (a) Snapshot of $u$ at $t \approx 3.5$, where $\Gamma$ is marked by a dashed line, (b) full data $u$ on $\Gamma$, (c) reference solution $u_{1}$, (d) the recovered $u_{1}^{h}$ obtained with $P=4$.

apply in the presence of inhomogeneity or obstacles compactly supported inside $\Omega_{1}$ or $\Omega_{2}$.

\section{Concluding remarks}

Starting from high-order ABC, we have shown how to split a wave field $u=u_{1}+u_{2}$ into individual "one-way" components $u_{1}$ and $u_{2}$, given observations of the total field $u$ at some recording boundary $\Gamma$. Thus, we can separate wave fields originating from different (unknown)
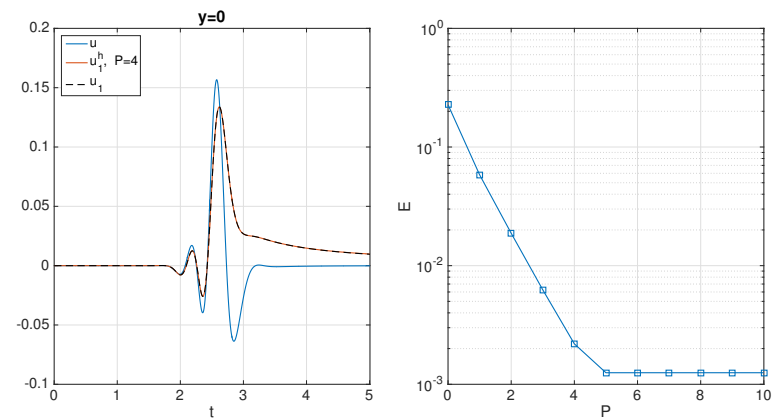

Figure 6: Echo removal. Left: the numerical solution $u$ driven by the two sources, the reference solution $u_{1}$ and the reconstructed signal $u_{1}^{h}$ at $(x, y)=(0,0)$; right: the relative global error $E$ as a function of $P$.

sources or remove an unwanted echo in measurements due to a (possibly unknown or moving) physical boundary. Our wave-splitting approach is deterministic and local both in space and time, as it involves no integrals or Fourier transforms. It also makes no prior assumptions on the frequency spectrum or the location of sources or physical boundaries.

To reconstruct $u_{1}$, for instance, we first choose a one-way differential operator, $B$, from a known high-order $\mathrm{ABC}$ for which $B\left[u_{2}\right]$ vanishes. Then, we numerically solve the IBVP (6) for $u_{1}$ in a computational domain, located on one side of $\Gamma$, where we impose an inhomogeneous boundary condition that involves the observations via $B[u]$. The accuracy in the reconstruction of $u_{1}$ (or $u_{2}$ ) is determined by the accuracy of the $\mathrm{ABC}$ and the numerical discretization error. Both can be made arbitrarily small and hence, in that sense, our approach is exact. As the computational domain along $\Gamma$ can be arbitrarily narrow, the computational effort for the reconstruction is kept minimal. While we have used the family of high- 
order ABCs by Collino [4] here, other ABCs certainly apply, too.

For inverse scattering problems, our approach also permits to extract the scattered field from measurements of the total (physical) wave field, when the incident wave is only partially known, say, when the location or direction but not the time dependence of the source is known. Even in situations of multiple scattering, our approach still permits to split the right- and left-moving components $u_{1}$ and $u_{2}$ of the total field bouncing back and forth between two obstacles. However, each individual wave field, $u_{i}$, will not precisely correspond to the scattered field resulting due to the illumination of a single obstacle, but instead also contain all higher order one-way moving multiple reflections.

\section{References}

[1] R. Aichner and H. Buchner and F. Yan and WKंellermann: A real-time blind source separation scheme and its application to reverberant and noisy acoustic environments., Signal Processing 86 (2006), pp. 1260-1277.

[2] A. Bayliss and E. Turkel: Radiation boundary conditions for wave-like equations. Comm. Pure Appl. Math. 33, pp. 707-725, 1980

[3] M. Castella, P. Bianchi, A. Chevreuil, J.-C. Pesquet: A blind source separation framework for detecting CPM sources mixed by a convolutive MIMO filter, Signal Processing 86 (2006), pp. 1950-1967.

[4] F. Collino, High Order Absorbing Boundary Conditions for Wave Propagation Models. Straight Line Boundary and Corner Cases,
Proc. 2nd Int. Conf. on Mathematical \& Numerical Aspects of Wave Propagation.

[5] B. Engquist and A. J. Majda: Absorbing boundary conditions for the numerical simulation of waves. Math. Comp. 31 (139), pp. 629-651, 1977

[6] D. Givoli, High-Order Local Non-Reflecting Boundary Conditions: A Review, Wave Motion, 39 (2004), 319-326.

[7] R. Griesmaier, M. Hanke and J. Sylvester, Far Field Splitting for the Helmholtz Equation, SIAM J. Numer. Anal. 52 (2014), pp. 343-362.

[8] M. J. Grote, C. Kirsch, Nonreflecting Boundary Conditions for Time-Dependent Multiple Scattering, J. Comput. Phys., 221 (2007), 41-62.

[9] M. J. Grote, M. Kray, F. Nataf and FÁssous, Time-dependent wave splitting and source separation, J. Comput. Phys., 330 (2017), 981-996.

[10] M. J. Grote: Local nonreflecting boundary condition for Maxwell's equations. Comput. Methods Appl. Mech. Engrg. 195, pp. 3691-3708, 2006

[11] T. Hagstrom and S. I. Hariharan, A formulation of asymptotic and exact boundary conditions using local operators, Appl. Numer. Math., 27, pp. 403-416 (1998).

[12] M. J. Grote, I. Sim, Perfectly Matched Layer for the Second-Order Wave Equation, Proceedings of the Ninth International Conference on Numerical Aspects of Wave Propagation (WAVES 2009, Pau, France, 2009), pp. 370-371. 
[13] T. Hagstrom, T. Warburton, Complete Radiation Boundary Conditions: Minimizing the Long Time Error Growth of Local Methods, SIAM J. Numer. Anal., Vol. 47 (2009), pp. 3678-3704.

[14] M. Pernot, G. Montaldo, M. Tanter, and M. Fink: Ultrasonic stars for time reversal focusing using induced cavitation bubbles, Appl. Phys. Lett. 88 (2006), pp. 034102

[15] R. Potthast, F. M. Fazi, and P. A. Nelson: Source splitting via the point source method, Inverse Problems 26 (2010), pp. 045002

[16] J. O. A. Robertsson, L. Amundsen: Wave equation processing using finite-difference propagators, Part 2: Deghosting of marine hydrophone seismic data, Geophysics, Vol. 79 (2014), pp. T301-T312.

[17] T. Saratoon, T. Tarvainen, B. T. Cox, and S. R. Arridge: A gradient-based method for quantitative photoacoustic tomography using the radiative transfer equation, Inverse Problems 29 (2013), pp. 075006

[18] L. N. Trefethen, L. Halpern, WellPosedness of One-Way Wave Equations and Absorbing Boundary Conditions, Math. Comp., Vol. 47 (1986), 421-435. 


\section{LATEST PREPRINTS}

No. Author: Title

2017-07 H. Harbrecht and M. Schmidlin

Multilevel Methods for Uncertainty Quantification of Elliptic PDEs with Random Anisotropic Diffusion

2017-08 M. Griebel and H. Harbrecht

Singular value decomposition versus sparse grids: Refined complexity

Estimates

2017-09 J. Garcke and I. Kalmykov

Efficient Higher Order Time Discretization Schemes for Hamilton-JacobiBellman Equations Based on Diagonally Implicit Symplectic Runge-Kutta Methods

2017-10 M. J. Grote and U. Nahum

Adaptive Eigenspace Regularization For Inverse Scattering Problems

2017-11 J. Dölz, H. Harbrecht, S. Kurz, S. Schöps and F. Wolf

A Fast Isogeometric BEM for the Three Dimensional Laplace- and

Helmholtz Problems

2017-12 P. Zaspel

Algorithmic patterns for $\mathcal{H}$-matrices on many-core processors

2017-13 R. Brügger, R. Croce and H. Harbrecht

Solving a free boundary problem with non-constant coefficients

2017-14 M. Dambrine, H. Harbrecht and B. Puig

Incorporating knowledge on the measurement noise in electrical impedance tomography

2017-15 C. Bürli, H. Harbrecht, P. Odermatt, S. Sayasone and N. Chitnis Analysis of Interventions against the Liver Fluke, Opisthorchis viverrini

2017-16 D. W. Masser

Abcological anecdotes

2017-17 P. Corvaja, D. W. Masser and U. Zannier

Torsion hypersurfaces on abelian schemes and Betti coordinates

2017-18 F. Caubet, M. Dambrine and H. Harbrecht

A Newton method for the data completion problem and application to obstacle detection in Electrical Impedance Tomography

2018-01 H. Harbrecht and P. Zaspel

On the algebraic construction of sparse multilevel approximations of elliptic tensor product problems 


\section{LATEST PREPRINTS}

No. Author: Title

2018-02 F. Ghiraldin and X. Lamy

Optimal Besov differentiability for entropy solutions of the eikonal equation

2018-03 H. Harbrecht and M. Schmidlin

Multilevel quadrature for elliptic problems on random domains by the coupling of FEM and BEM

2018-04 M. Bugeanu and H. Harbrecht

Parametric representation of molecular surfaces

2018-05 A. Abdulle, M. J. Grote and O. Jecker

Finite element heterogeneous multiscale method for Elastic Waves in Heterogeneous Media

2018-06 M. J. Grote and J. H. Tang

On controllability methods for the Helmholtz equation

2018-07 H. Harbrecht and M. Moor

Wavelet boundary element methods - Adaptivity and goal-oriented error estimation

2018-08 P. Balazs and H. Harbrecht

Frames for the solution of operator equations in Hilbert spaces with fixed dual pairing

2018-09 R. Brügger, R. Croce and H. Harbrecht

Solving a Bernoulli type free boundary problem with random diffusion

2018-10 J. Dölz, H. Harbrecht and M. D. Multerer

On the best approximation of the hierarchical matrix product

2018-11 H. Harbrecht and P. Zaspel

A scalable $\mathcal{H}$-matrix approach for the solution of boundary integral equations on multi-GPU clusters

2018-12 H. Harbrecht, N. Ilić and M. D. Multerer

Acoustic scattering in case of random obstacles

2018-13 D. H. Baffet, M. J. Grote, S. Imperiale and M. Kachanovska

Energy decay and stability of a perfectly matched layer for the wave equation

2018-14 D. Baffet and M. J. Grote

On wave splitting, source separation and echo removal with absorbing boundary conditions 